\title{
Commentary: Is it Time To Reexamine our Policies?
}

T. Allen Merritt, MD, MHA, Mitchell Goldstein, MD

In June 2020, Neonatology Today summarized recommendations from the US, Ireland, and the UK regarding the management of mothers with or at a very high risk for COVID-19 infection and care for their infants. During the following past four months, data from COVID-19 "hot spots" in New York has shed new light on the risk of vertical transmission among a cohort of newborns whose mothers had confirmed or suspected SAR-CoV-2 infection as reported by Gyamfi-Bannerman et al. from Columbia University Irving Medical Center in New York City (1). Among 101 newborns, there was no detection of SARS-CoV-2 viral RNA in 95.7\% of nasopharyngeal $\mathrm{PCR}$ tests for an overall transmission rate of $2 \%$. Of the two infants who did have evidence of vertical transmission, both had indeterminate tests. The authors conclude that they were infected with very low viral copy numbers, and neither newborn has symptoms.

"During the following past four months, data from COVID-19 "hot spots" in New York has shed new light on the risk of vertical transmission among a cohort of newborns whose mothers had confirmed or suspected SAR-CoV-2 infection as reported by Gyamfi-Bannerman et al. from Columbia University Irving Medical Center in New York City (1)."

Notably, about $75 \%$ of mothers were roomed-in with their newborns, while six infants were separated from severely ill mothers cared for in the ICU, and 19 infants were admitted to the NICU without contact with their parents. The authors conclude, "This study endorses the benefits of rooming-in, establishing breastfeeding, and delaying pathing on newborn outcomes and suggests that separating mothers positive for SARS-CoV-2 and their newborns and avoiding direct breastfeeding may not be warranted to present SARS-CoV-2 transmission." The American Academy of Pediatrics Perinatal COVID-19 Registry data from more than 3000 deliveries also suggests that about $2 \%$ of infants born to women who test positive for SARS-CoV-2 near the time of delivery have tested positive (using PCR testing) within four days of birth. These studies suggest that the vast majority of newborns who are asymptomatic shortly after birth are unlikely to have had a vertical transmission of the virus.

Although the timing of maternal infection in most cases was indeterminant, given this very low risk of postnatal infection, the policy of maternal-newborn separation needs to be reexamined; however, infection control measures still need to be rigorously applied. The Columbia study collected maternal, and infant paired samples admitted from March 13 to April 14 from New York-Presbyterian Morgan Stanley Children's Hospital and New York-Presbyterian Allen Hospital. All infants included in this study underwent SARSCoV-2 testing on their first day of life, and several had multiple tests during the nursery stay.
Ninety percent of women who were infected were asymptomatic or had a mild illness. The median maternal age was 28.5 years and over one-third has higher risk, including asthma, diabetes, chronic hypertension, or pregnancy-associated hypertension. Over $50 \%$ had BMIs $>30$. Newborns delivered of mothers with severe or critical COVID-19 infections were significantly more likely to be born 1.4 weeks prior to the expected date of delivery $(P<0.02)$, and these infants were at higher risk for hyperbilirubinemia requiring phototherapy $(p<0,04)$. One newborn with evidence of vertical transmission was delivered vaginally and underwent testing on day three after birth, while the other was delivered by cesarean section, and the mother was asymptomatic. The authors caution that their findings were from a pandemic epicenter and may not be generalizable to other communities, and most of the women were likely infected in their third trimester.

"The American Academy of Pediatrics Perinatal COVID-19 Registry data from more than 3000 deliveries also suggests that about $2 \%$ of infants born to women who test positive for SARS-CoV-2 near the time of delivery have tested positive (using PCR testing) within four days of birth. These studies suggest that the vast majority of newborns who are asymptomatic shortly after birth are unlikely to have had a vertical transmission of the virus."

Given these limitations, neonatologists and pediatricians may need to balance risks of mother/infant separation, and an accompanying editorial (2) also stresses that careful assessment of mothers and infants needs to continue, but given the low rate of vertical transmission reported, prior recommendations should be carefully considered and modified.

"Given these limitations, neonatologists and pediatricians may need to balance risks of mother/infant separation, and an accompanying editorial (2) also stresses that careful assessment of mothers and infants needs to continue, but given the low rate of vertical transmission reported, prior recommendations should be carefully considered and modified." 
References:

1. Dumitriu D, Emenuwa, U, Hanft E et al. Outcomes of Neonates Born to Mothers with Severe Acute Respiratory Syndrome Coronavirus 2 infection at a Large Medical Center. JAMA Pediatr October 12, 2020 dol: 10.1001/jamapediatrics. 2020.4298

2. Kimberline $D$ and Puopolo K. "Balancing risks in the time of COVID-19" JAMA Pediatr October 12, 2020. Doi 10.1001/ jamapediatrics.2020.4304.

The author has no conflicts to disclose

\section{NT}

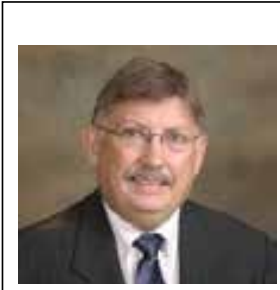

Corresponding Author

T.Allen Merritt, MD

Professor of Pediatrics

Loma Linda University School of Medicine

Division of Neonatology

Department of Pediatrics

email: T. Allen Merritt <allenmerritt.md@gmail.com>

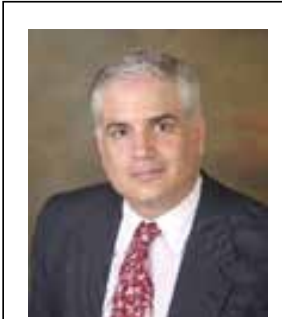

Mitchell Goldstein, MD

Professor of Pediatrics

Loma Linda University School of Medicine

Division of Neonatology

Department of Pediatrics

mgoldstein@llu.edu

\section{Readers can also follow NEONATOLOGY \\ via our Twitter Feed \\ @NEO}

\section{Visit us at www.mynicunetwork.org}

\section{Education that is...}

- Story-Driven

- Trauma-Informed

- Evidence-Based

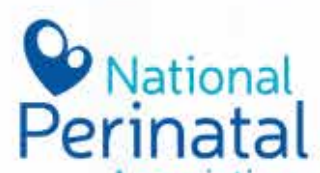
Association

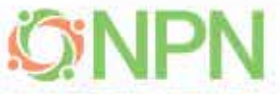

NICU PAFENT NETWORK

The preeminent provider of compelling perinatal education on

psychosocial support created through interprofessional collaboration 